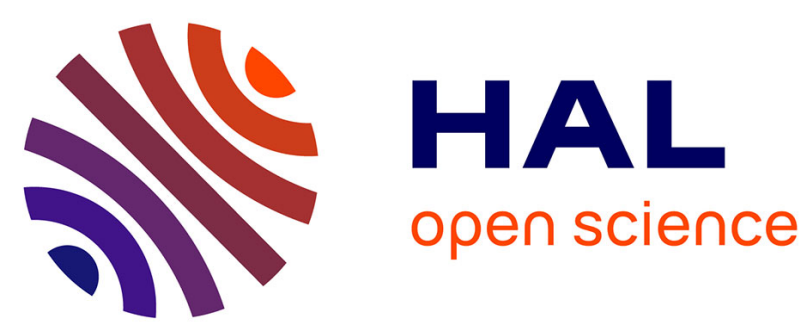

\title{
Defect sizing using convolution neural network applied to guided wave imaging
}

Andrii Kulakovskyi, Olivier Mesnil, Bastien Chapuis, Oscar D'almeida, Alain Lhémery

\section{- To cite this version:}

Andrii Kulakovskyi, Olivier Mesnil, Bastien Chapuis, Oscar D'almeida, Alain Lhémery. Defect sizing using convolution neural network applied to guided wave imaging. Structural Health Monitoring 2019, Oct 2019, Stanford, United States. 10.12783/shm2019/32195 . hal-03246897

\section{HAL Id: hal-03246897 https://hal.science/hal-03246897}

Submitted on 2 Jun 2021

HAL is a multi-disciplinary open access archive for the deposit and dissemination of scientific research documents, whether they are published or not. The documents may come from teaching and research institutions in France or abroad, or from public or private research centers.
L'archive ouverte pluridisciplinaire HAL, est destinée au dépôt et à la diffusion de documents scientifiques de niveau recherche, publiés ou non, émanant des établissements d'enseignement et de recherche français ou étrangers, des laboratoires publics ou privés. 
Title: Defect sizing using convolution neural network applied to guided wave imaging

\author{
Authors : Andrii Kulakovskyi \\ Olivier Mesnil \\ Bastien Chapuis \\ Oscar d'Almeida \\ Alain Lhémery
}




\begin{abstract}
The lightweight aluminum alloys are extensively used in the aerospace industry. These materials are used for constructing complex structures such as aircraft fuselage due to their excellent strength-to-weight ratio, stiffness, and corrosion resistance. However, defects such as corrosion or fractures can appear because of thermo-mechanical aging in a hostile working environment or impact forces due to the improper use of these structures. In light of this, Guided Waves (GWs)-based Structural Health Monitoring (SHM) system can be considered as a promising solution for the structural integrity screening, maintenance costs reduction and prolongation of the service time of these materials.

In general, a sparse array of PZT transducers can be used for GWs exciting and sensing, and GWs Imaging (GWI) algorithms, such as Excitelet, can be used to process the measured signals. This imaging technique allows computing high-resolution images that represent the integrity of the structure, where each pixel of the image is mapped to the elementary portion of the structure and carries a Damage Index (DI) value. While defect presence and location can be determined from visual inspection of the image by naked eye, the defect sizing is a more complex problem due to non-linear behaviour of DI values regarding the defect size and its location.

This paper proposes an approach for defect size evaluation. It relies on the extensive GWI database generated by means of Spectral Finite Elements modelling method implemented in CIVA and Convolution Neural Network (CNN) trained on numerical data. The CNN is used to build an accurate inversion model that takes GWI sample as input and determines the size of the defect. The model is tested on the simulated data and validated by means of experiment in aluminum plate.
\end{abstract}

\title{
INTRODUCTION
}

One of the most important challenges for engineering structure manufacturers, endusers, and maintenance teams is to evaluate the integrity of in-service structures on a frequent or continuous time basis. Applied physical loads, thermo-mechanical aging,

Andrii Kulakovskyi, Oscar d'Almeida, Safran SA, TCA, Châteaufort, Yvelines, France Olivier Mesnil, Bastien Chapuis, Alain Lhémery, CEA LIST, Gif-sur-Yvette, France 


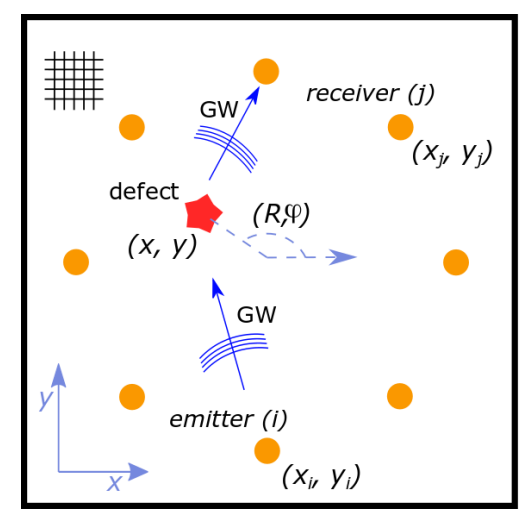

Figure 1: Illustration of GWI methodology. Yellow circles and the red star denote locations of PZTs and structural flaw, respectively. Blues arrows represent GWs propagating in the structure.

impacts and other external factors deteriorate their mechanical properties and might lead to defects. Different sectors, the aerospace industry in particular, are concerned with this problematic.

Aforementioned reasons became a driving force for the development of structural health monitoring system (SHM) to provide an accurate localization and quantification of damages in engineering structures while being robust to the environmental effects such as temperature and humidity variations, accumulated strains, etc [1,2]. SHM relies on permanently embedded transducers and knowledge-based methods, i.e., signal processing algorithms. Embedded transducers are typically used for measuring a structural dynamic response either in a passive or active way. A knowledge-based system then processes the collected signals in order to evaluate the current health of the structure [3]. SHM system can potentially replace scheduled maintenance, and lead to a reduction of safety design margin, hence introduce significant economic benefit.

Guided elastic waves (GW) have been proven effective for structure inspection as they can propagate over large distances while being sensitive to structural defects [4]. Among other SHM methods, GWs can be easily actuated and sensed by a network of piezoelectric transducers (PZTs). At any excitation frequency, at least two fundamental modes are generated and propagated with different velocities. Hence, measured signals are complex, and typically contain multiple overlapping wavepackets that correspond to incident GWs as well as their boundary reflections. GWs are also dispersive, so the shape of wavepackets changes with propagating distance. Both phenomena make interpretation of GWs signals difficult and require sophisticated methods for processing.

Among different categories of GWs processing methods, the Guided Waves Imaging (GWI) is standing out. GWI is an effective approach to evaluate the integrity of the structure. It implies an image computation for the region of interest of the studied specimen, where each pixel is mapped to the corresponding segment of the structure and carries a DI value.

In the current work, the defect imaging is performed by means of a correlation-based algorithm named Excitelet [5]. For each pixel of the image, it computes DI values as a correlation coefficient between theoretical and experimental GW signals. While the single DI value does not provide sufficient information about the integrity of the structure, 


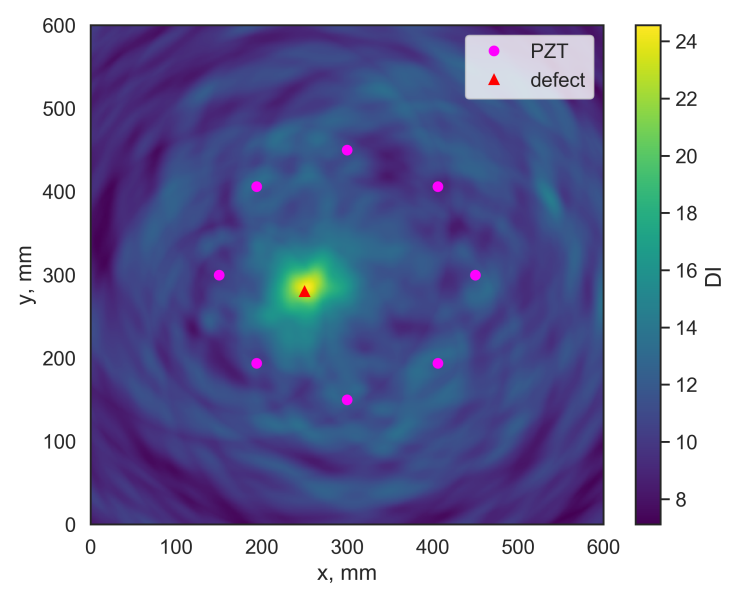

Figure 2: Example of experimental GWI result in aluminum plate $3 \mathrm{~mm}$ thick where the defect is represented by the transverse hole $10 \mathrm{~mm}$ in diameter. The GWI is performed using Excitelet algorithms at $45 \mathrm{kHz}$.

the spatial distribution of DI values can be interpreted for determining the presence of the defect, its location [6]. The damage detection and localization are straightforward from the image analysis, whereas damage sizing is a more complex problem that might require sophisticated solvers including machine learning methods.

This paper presents a data-driven approach for defect size evaluation by means of CNN. Its is organized as follows. Firstly, a brief review of GWI principle and Excitelet defect imaging algorithms is presented. Then, inversion problem of defect sizing from GWI samples is formulated. An extensive database of simulated GWI results is generated and processed by means of a $\mathrm{CNN}$ in order to construct an inversion model capable of determining the size of a defect from images. Finally, an inversion model is tested on numeric and validated on experimental GWI data.

\section{GUIDED WAVES IMAGING}

A number of research works, Wang et al. [7] and Michaels et al. [8] in particular, have demonstrated that information from multiple transducers can be processed and combined in order to compute an image that represents the integrity of the structure. Main advantages of GWI approach are its robustness to signal corruption and that it provides visually interpretable results as compared to non-imaging methods.

A schematic of the GWI procedure is represented in Figure 1. The grid of pixels discretizes the region of interest of the studied specimen. As mentioned previously, we use a baseline demanding algorithm Excitelet [5]. For each pixel of the image, it attributes DI values by computing a correlation coefficient between experimental residual signals (defined as the difference between pristine and damaged states of the structure) and theoretical signals that represent dispersed versions of the excitation signal. Theoretical signal can be expressed as a convolution between excitation function and 3D Green's function of the medium. The latter can be obtained by means of GWs simulation frameworks such as SAFE [9].

As the GWs dispersion is taken into consideration, the structure can be excited over a 


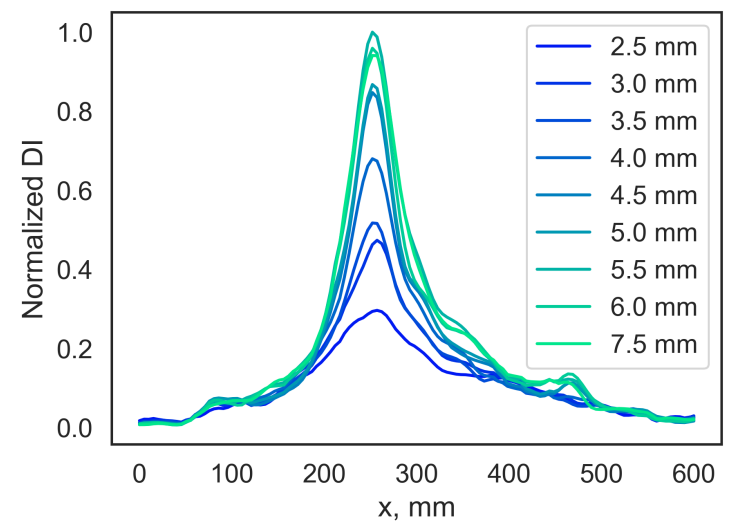

Figure 3: Normalized through defect DI values profiles.

large bandwidth, which allows processing a damage response in a large frequency range. The DI value is computed as follows:

$$
D I^{E X C}(x, y)=\sum_{i=1}^{N-1} \sum_{j=i+1}^{N}\left|\frac{\int u_{i, j}(t) s_{i, j}(x, y, t) d t}{\left|\int u_{i, j}(t) d t\right|\left|\int s_{i, j}(x, y, t) d t\right|}\right| .
$$

It should be mentioned that Excitelet presumes imaging of a point-like defect, so the wavelength of inspecting GW should be at least twice larger than the defect size.

\section{INVERSION PROBLEM FORMULATION}

GWI as such is an effective method and a natural choice for a GWs-based SHM system. Here, the studied GWI configuration is the aluminum plate $600 \mathrm{~mm} \times 600 \mathrm{~mm}$ $\times 3 \mathrm{~mm}$ in dimension. The plate is equipped with eight PZT transducers $9 \mathrm{~mm}$ in radius and the defect is represented by the transverse hole. The corresponding GWI result for hole $5 \mathrm{~mm}$ in radius located at $(X: 250, Y: 280, \mathrm{~mm})$ is shown in Figure 2. It can be observed that the damage detection and localization are straightforward from the visual analysis of the intensity distribution in image, whereas damage sizing is not trivial. Consider the same GWI configuration. The structural damage is represented by a transverse hole located at $(X: 250, Y: 280, \mathrm{~mm})$ that progressively grows from $2.5 \mathrm{~mm}$ to $8.5 \mathrm{~mm}$ in radius with increment of $0.5 \mathrm{~mm}$. The plate is excited with two-cycle Hanning modulated tone burst, and the defect imaging is performed by Excitelet algorithm using $A_{0}$ mode where $\lambda_{A_{0}}^{45 \mathrm{kz}}=24.85$, mm. Corresponding GWI results are normalized with respect to the highest DI value, and DI profiles through the flawed region are shown in Figure 3. It can be observed that for small defects $(R \subset[2.5,5.5], \mathrm{mm})$ DI values increase progressively and sharpen with the radius of the hole, but for larger ones they saturate and decrease in magnitude.

The DI distribution complies with the bivariate Gaussian distribution. Therefore, the maximum DI (max-DI) values and corresponding through defect Full Width Half Maximum (FWHM) in both $X$ and $Y$ directions can be collected for the sake of demonstration of GWI sensitivity to the defect size, see Figure 4. It is worth noting that there are regions where dependence is almost linear $(D I \subset[2.5,4.5], \mathrm{mm}$ and $D I \subset[6.0,8.5], \mathrm{mm})$ 


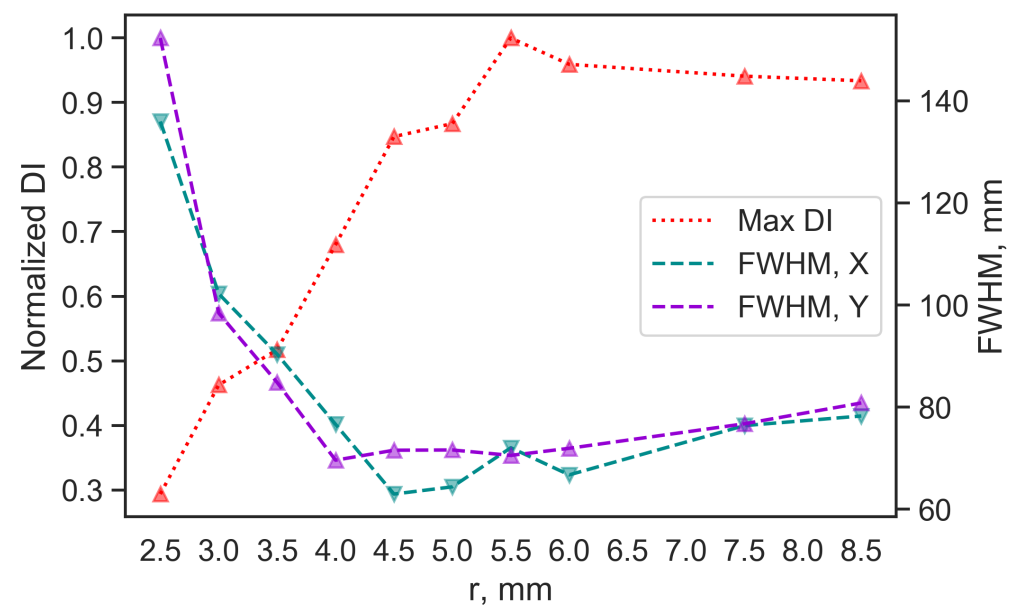

Figure 4: Normalized Max-DI and FWHM versus hole radius.

but, in total, these variations are non-linear in both height and width. It should also be pointed out that DI values distribution is not spatially homogeneous, meaning that DI values differ for the same size defects located at various positions.

Taking into account such a non-linear behavior of DI values distribution regarding the defect location and size variations, the analytical solution for a defect sizing problem is not straightforward. Therefore, a data-driven approach is proposed to search for a solution to this problem as it is capable of approximating such complex non-linear functions. Consider a data set of $N$ elements $D \equiv\left\{x_{i}, y_{i}\right\}_{i=1}^{N}$, where $x$ and $y$ denote GWI results and corresponding defect sizes. The data-driven approach is aimed at learning structural relations in $D$ in order to derive an image inversion model $f(x)$ so that this model would be capable of determining a defect size $y_{*}$ by processing any new image $x_{*}$ for a given GWI configuration. More formally, it can be expressed as:

$$
y=f(x)+\epsilon,
$$

where $f(x)$ is non-linear the model that maps images $x$ to the corresponding size $y$ and $\epsilon$ represents the independent noise. The approximation of the model $f(x)$ can be obtained using machine learning methods and is presented in the following section.

\section{DEEP LEARNING FOR DEFECT SIZE INVERSION}

Nowadays, deep learning (DL) is of great interest in many fields. This is a subfield of machine learning that uses multi-layered Artificial Neural Networks (ANNs) for completing challenging tasks such as image processing, object detection, speech recognition, language translation and many others [10]. Its recent success can be largely attributed to the strong emphasis on modeling multiple levels of abstractions with the depth of the DL model: from low-level features to higher-order representations using multiple, and usually non-linear, transformations. Convolution Neural Network [11] is one of the many possible DL architectures that have been proven particularly effective for image analysis, and, therefore, has been selected for GWI result processing. 


\section{Introduction to Convolution Neural Network}

Convolution neural network $(\mathrm{CNN})$ is a major breakthrough in the field of deep learning. Numerous practical machine learning tasks, such as image classification, object recognition, control-policy learning for autonomous ground and aerial vehicles $[12,13]$ or the board game Go are successfully solved using CNNs.

This type of DL models searches for patterns in images, determines relevant features for the current problem and consequently adapts persecutors. CNNs are typically composed of multiple aggregated layers, each computes convolution transforms, applies non-linear activations and pooling operators as it goes deep [14,15]. Aggregated layers are sequentially stacked and connected to fully connected layers. A set of convolution operators, also called kernels, forms a convolution layer. For a given position, a kernel outputs a high value if the convolution feature is present in that position, otherwise the output is low. The main purpose of kernels is to capture spatially dependent information, i.e. features, in the input. It is worth noting that a convolution layer typically may consist of dozens and even hundreds of kernels. Convolution operators are followed by the application of an activation function. Perhaps, the most prominent and effective activation function for CNNs is a Leaky Rectified Linear Unit (1-ReLU) [16], as it allows overcoming efficiently the vanishing gradient problem [17].

The last operator of an aggregated convolution layer is a pooling, i.e. subsampling, operator [18]. It is mainly used to decrease the variance and reduce computational complexity of the activation map. In this work, the average pooling is used as it allows extracting smooth features.

Aggregated convolution layers are connected to fully-connected layers. The term fullyconnected implies that each neuron in the previous layer is connected to every neuron in the next layer. Therefore, high-level features of the input image learned by previous convolution and pooling layers can be combined so that the network can build the global representation of the entire image [19]. Adding several layers to the neural network allows learning features in hierarchical order, and their level of abstraction progressively grows with the depth of the model. In general, the performance of a neural network increases with the number of layers, but deeper models are more difficult to train and require a larger amount of data.

If the network is differentiable, the network training, i.e., kernel tuning, can be performed using the back propagation algorithm [18]. The back propagation method allows adjusting kernels proportionally to their contribution to the total output error [20].

\section{Model training}

In general, data-driven methods, and CNN in particular, require a large amount of data to construct an accurate model. Indeed, an experimental database is desired because it is fully representative and reproduces operational noise, which is inherent to the GW-based SHM process. However, the generation cost of the experimental database is prohibitive as a large number of samples are required. Therefore, a Spectral Finite Elelement (SFE) simulation method $[21,22]$ is used in this work to generate a dataset of GWI samples.

The GWI configuration is the same as previously described. Each simulated sample 
contains a transverse hole of varying size and position. Holes are distributed within the circle of transducers. Damage radii vary from $2.5 \mathrm{~mm}$ to $7.5 \mathrm{~mm}$ and comply with random uniform distribution. Excitation function is a two-cycle Hanning modulated tone burst centered at $45 \mathrm{kHz}$. In total, 917 SFEM simulations are run to generate a dataset of GWs signals, and Excitelet algorithm is then applied to produce a GWI database.

Each image in a GWI dataset is normalized and then cropped in order to preserve only the inner circle area. Afterwards, the dataset is randomly shuffled and split into two parts: training and testing sub-sets. The first one contains 779 images and the second one consists of 139 images. It is worth noting that images in a training sub-set are arranged in randomly shuffled batches, each contains 32 GWI samples due to computational reasons. In total, 1000 training epochs are run during the training phase and the performing model is selected among the proposed candidates. From now on, GWI samples are denoted as inputs and reference defect radii as outputs or target values, respectively. In this work, CNN's architecture has been manually tuned and regularized to achieve the best performance and to find a balance between overfitting and underfitting.

Thereby, the CNN is constructed as follows. It consists of three aggregated convolution layers, each contains 16, 32 and $64(3 \times 3)$ convolution kernels that slide over the image with the stride 1 . The activation function is 1-ReLU with $\alpha=0.3$ and the averagepolling kernels are of the size $(2 \times 2)$. Aggregated convolution layers are connected to two sequential fully connected layers that allow combining all the learned patterns. The first fully connected layer contains 128 neurons and the second one - 64 .

Here, the CNN is used to build a defect size inversion model from the non-linear regression perspective. Therefore, the root mean square (RMS) metric is used to evaluate a model's accuracy during training and testing phases. Neuron weights are updated using Adaptive Moment Estimation (ADAM) optimizer [23].

\section{Defect inversion results}

Transverse hole sizing results on both numerical and experimental data are presented in Figure 5, where the blue dashed line denotes reference values, red dots represent inversion results of simulated GWI samples, and green triangles represent experimental transverse hole inversion results. Accuracy metrics such as mean absolute error (MAE), standard deviation (STD), maximum absolute sizing error (max-ASE) and R2-score are evaluated in order to estimate the performance of the CNN. The first three metrics are straightforward from the term, and R-squared (R2 score) describes the fraction by which the variance of ASEs is less than the variance of reference defect radii, where the best possible value of R2 score is one. It is worth noting that the testing sub-set contains GWI samples computed for different defect sizes at various locations, therefore, performance metrics evaluated on the testing dataset can be generalized and considered as fully representative for the current inversion model.

CNN demonstrates a relatively good performance in determining radii of defects from simulated GWI results. The MAE is equals to $0.32 \mathrm{~mm}$, the STD is $0.3 \mathrm{~mm}$, the R2 score is $0.89 \mathrm{~mm}$, and max-ASE is $1.65 \mathrm{~mm}$. It is worth mentioning that experimental GWI results were only normalized, meaning that no filtering or preprocessing have been applied - they are used as such. Defect size inversion results are plotted against their

reference values and overall $\mathrm{CNN}$ predictions from other defect locations, see Figure 


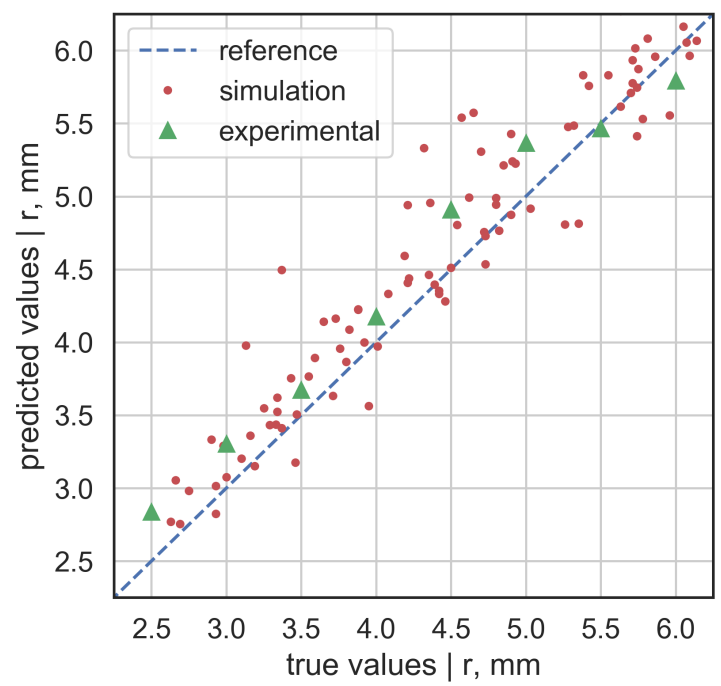

Figure 5: Inversion of experimental GWI results at $45 \mathrm{kHz}$.

5. It can be seen that radii predictions of experimental defects are accurately estimated by the model and their spread remains in the limits of the model's uncertainty. The submillimeter defect sizing accuracy for both simulated and experimental GWI results is achieved using images computed with spatial step of $5 \mathrm{~mm}$, where the wavelength of the $A_{0}$ mode at $45 \mathrm{kHz}$ is of about $25 \mathrm{~mm}$. One of the driving factors of such precision is that the CNN is capable of perceiving not only single DI values but their mutual spatial distribution.

\section{CONCLUDING REMARKS}

This paper presents an approach for the defects' size evaluation using GWI results. GWI is a robust and effective method for GWs signals processing that allows computing images representing the integrity of the structure. Defect detection and localization are straightforward from the analysis of these images, but defect sizing is a more complex problem. There are two main difficulties associated with this problem. First, the spatial intensity distribution of these images for the defects of different sizes that are located at the same position varies in non-linear manner. Second, the spatial intensity distribution depends on the location for defects of the same size. Therefore, a data-driven approach is proposed for defect sizing as it allows determining an approximate solution for complex problems, including the above mentioned one.

The proposed approach is based on the use of a CNN. Among different data-driven approaches, this one is particularly interesting as it allows capturing both local and global spatial relations between the pixels of the image. The flexibility of the model and the degree of abstraction of learned patterns are controlled by the depth and width of the model, whereas the balance between overfitting and underfitting is kept by the regularization. As the name suggests, any data-driven method, including this one, requires a large amount of data to build a well performing model. The SFE method and Excitelet algorithm are used for generating an extensive database of GWI samples. This dataset is 
created for a specific GWI configuration but represents defects of different sizes at various locations. Each GWI sample is normalized in order to unify simulated and experimental results that are used for validation. It is worth noting that the simulated dataset does not contain a GWI sample corresponding to the experiment.

The developed defect sizing model is validated on both unseen simulated and experimental GWI results and demonstrates a good performance. The proposed approach allows completing the GW-based SHM system pipeline so that the defect detection, localization and quantification are possible. For future work, authors will focus on development of the defect sizing model for composite materials under varying GWI configurations and taking into consideration environmental effects.

\section{REFERENCES}

1. Su, Z., L. Ye, and Y. Lu. 2006. "Guided Lamb waves for identification of damage in composite structures: A review," Journal of Sound and Vibration, 295(3-5):753-780.

2. Diamanti, K. and C. Soutis. 2010. "Structural health monitoring techniques for aircraft composite structures," Progress in Aerospace Sciences, 46(8):342-352.

3. Giurgiutiu, V. 2014. Structural Health Monitoring with Piezoelectric Wafer Active Sensors, Elsevier Academic Press.

4. Sohn, H., C. R. Farrar, N. F. Hunter, and K. Worden. 2001. "Structural health monitoring using statistical pattern recognition techniques," Journal of dynamic systems, measurement, and control, 123(4):706-711.

5. Quaegebeur, N., P. Masson, D. Langlois-Demers, and P. Micheau. 2011. "Dispersion-based imaging for structural health monitoring using sparse and compact arrays," Smart Materials and Structures, 20(025005).

6. Kulakovskyi, A., B. Chapuis, O. Mesnil, N.-R. Bedreddine, O. d'Almeida, and A. Lhmery. 2017. "Defect imaging on CFRP and honeycomb composite structures by guided waves generated and detected by a sparse PZT array," in Proceedings of the 11th International Workshop on Structural Health Monitoring.

7. Wang, C. H., J. T. Rose, and F.-K. Chang. 2004. "A synthetic time-reversal imaging method for structural health monitoring," Smart Materials and Structures, 13(2):415.

8. Michaels, J. E. 2008. "Detection, localization and characterization of damage in plates with an in situ array of spatially distributed ultrasonic sensors," Smart Materials and Structures, 17(035035).

9. Velichko, A. and P. D. Wilcox. 2007. "Modelling the Excitation of Guided Waves in Generally Anisotropic Multi-layered Media," Journal of the Acoustical Society of America, 121(60).

10. LeCun, Y., Y. Bengio, and G. Hinton. 2015. "Deep learning,” Nature, 521(7553):521.

11. Szegedy, C., V. Vanhoucke, S. Ioffe, J. Shlens, and Z. Wojna. 2015. "Rethinking the Inception Architecture for Computer Vision," ArXiv: Computer Vision and Pattern Recognition.

12. Maei, H. R., C. Szepesvari, S. Bhatnagar, and R. S. Sutton. 2010. "Toward O-Policy Learning Control with Function Approximation," in ICML.

13. Zhang, T., T. G. Kahn, S. Levine, and P. Abbeel. 2016. "Learning deep control policies for autonomous aerial vehicles with mpc-guided policy search," in IEEE international conference on robotics and automation (ICRA), pp. 528-535.

14. Szegedy, C. ., W. Liu, Y. Jia, P. Sermanet, S. Reed, D. Anguelov, D. Erhan, V. Vanhoucke, and A. Rabinovich. 2015. "Going deeper with convolutions," in In Proceedings of the IEEE conference on computer vision and pattern recognition, pp. 1-9. 
15. Wiatowski, T. and H. Bolcskei. 2018. "A Mathematical Theory of Deep Convolutional Neural Networks for Feature Extraction," IEEE Transactions on Information Theory, 64:18451866.

16. Shang, W., K. Sohn, D. Almenia, and H. Lee. 2016. "Understanding and Improving Convolutional Neural Networks via Concatenated Rectified Linear Units," in International Conference on Machine Learning, pp. 2217-2225.

17. Maas, A., H. Y. Awni, and A. Ng. 2013. "Rectifier nonlinearities improve neural network acoustic models," in Proceedings of the 30-th International Conference on Machine Learning, vol. 28, p. 3.

18. Krizhevsky, A., I. Sutskever, and G. E. Hinton. 2012. "ImageNet Classification with Deep Convolutional Neural Networks," in F. Pereira, C. J. C. Burges, L. Bottou, and K. Q. Weinberger, eds., Advances in Neural Information Processing Systems, Curran Associates, Inc., pp. 1097-1105.

19. Simonyan, K. and A. Zisserman. 2014. "Very deep convolutional networks for large-scale image recognition," ArXiv: Computer Vision and Pattern Recognition.

20. Goodfellow, I., Y. Bengio, and A. Courville. 2016. Deep Learning, MIT Press.

21. Mesnil, O., A. Imperiale, E. Demaldent, V. Baronian, and B. Chapuis. 2018. "Simulation tools for guided waves based structural health monitoring," in AIP Conference Proceedings.

22. Mesnil, O., A. Imperiale, E. Demaldent, and B. Chapuis. 2018. "Validation of spectral finite element simulation tools dedicated to structural health monitoring," in AIP Conference Proceedings.

23. Kingma, D. P. and J. Ba. 2014. "Adam: A method for stochastic optimization," ArXiv: Machine Learning. 